\title{
Macrocyclic Versus Linear Gadolinium Chelates
}

\author{
Val M. Runge, $M D$
}

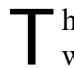
he article "High signal intensity in the dentate nucleus and globus pallidus on unenhanced T1weighted images: evaluation of the macrocyclic gadolinium-based contrast agent gadobutrol" by Radbruch et $\mathrm{al}^{1}$ provides critical new information regarding gadolinium deposition in the dentate nucleus. The conclusion, from a study of 30 patients, is that no signal intensity increase is seen with gadobutrol. This is in sharp distinction to a prior publication ${ }^{2}$ and, as stated in the conclusion of Radbruch et al, "adds further support to the hypothesis that the molecular structure of a gadolinium based contrast agent (GBCA) as either macrocyclic or linear is the crucial factor for its potential to cause gadolinium deposition in the brain."

History is important, in particular, to avoid the mistakes of the past. Publication and dissemination of well-performed, scientifically valid research are also crucial. Unfortunately, the scientific literature contains errors, many accidental, which in most cases are corrected with time. A prominent example was the belief that peptic ulcers were caused by acid. Indeed, this was part of my instruction, along with contemporaries across the world, while at Stanford Medical School in the late 1970s. It took years of dedicated research by Barry Marshall and Robin Warren, ${ }^{3}$ beginning in 1983, to dispel this belief. Because of their work, which was awarded a Nobel Prize in 2005, treatment today is simple and effective, with the enormous costs (both financial and in human terms) of surgery and lifelong medical treatment so avoided.

Unfortunately, scientific fraud also occurs, although rare. In the radiology literature, the best known example is that of Dr Robert A. Slutsky at the University of California San Diego in the 1980s. Of the 137 articles published by Dr Slutsky (over only a 6-year period), it was eventually concluded that 77 were valid, 48 were questionable, and 12 were fraudulent. ${ }^{4}$ Yet, despite the attention that this case received, even articles that were retracted continued to be cited.

Regarding the publication in European Radiology $y^{2}$ that the current results call into question, it is important to note that the European Radiology study was previously rejected by Investigative Radiology. A decision on rejection was reached in part due to the only figure provided not showing an increase in dentate nucleus signal intensity and the absence of a control patient population.

Incorrect conclusions drawn on the basis of clinical studies, regarding specifically the dentate nucleus T1-hyperintensity issue, likely also include 2 studies — published in 2009 and 2011 - that attribute the T1-hyperintensity of the dentate nucleus to secondary progressive multiple sclerosis ${ }^{5}$ and brain irradiation. ${ }^{6}$ Looking at this issue in retrospect, both patient populations are ones in which a high number of gadolinium chelate administrations would have been likely, thus arguing that the findings were likely not related to the disease process but rather to multiple prior gadolinium chelate administrations.

The study of Radbruch et al provides further evidence supporting a significant difference between macrocyclic and linear chelates in terms of safety profile. As the authors state in their conclusion, for linear GBCAs - specifically those that have no benefit in terms of improved diagnosis in comparison to the approved macrocyclic GBCAs - their ongoing clinical use should be reassessed. Let us remember, as physicians, above all to do no harm.

\section{REFERENCES}

1. Radbruch A, Weberling LD, Kieslich PJ, et al. High signal intensity in the dentate nucleus and globus pallidus on unenhanced T1-weighted Images: evaluation of the macrocyclic gadolinium-based contrast agent gadobutrol. Invest Radiol. 2015;50:805-810.

2. Stojanov DA, Aracki-Trenkic A, Vojinovic S, et al. Increasing signal intensity within the dentate nucleus and globus pallidus on unenhanced T1W magnetic resonance images in patients with relapsing-remitting multiple sclerosis: correlation with cumulative dose of a macrocyclic gadolinium-based contrast agent, gadobutrol. Eur Radiol. 2015 [Epub ahead of print].

3. Marshall BJ, Warren JR. Unidentified curved bacilli in the stomach of patients with gastritis and peptic ulceration. Lancet. 1984;1:1311-1315.

4. Whitely WP, Rennie D, Hafner AW. The scientific community's response to evidence of fraudulent publication. The Robert Slutsky case. JAMA. 1994;272:170-173.

5. Roccatagliata L, Vuolo L, Bonzano L, et al. Multiple sclerosis: hyperintense dentate nucleus on unenhanced T1-weighted MR images is associated with the secondary progressive subtype. Radiology. 2009;251:503-510.

6. Kasahara S, Miki Y, Kanagaki M, et al. Hyperintense dentate nucleus on unenhanced T1-weighted MR images is associated with a history of brain irradiation. Radiology. 2011;258:222-228.

Received for publication September 21, 2015; and accepted for publication, after revision, October 2, 2015.

From the Department of Diagnostic, Interventional and Pediatric Radiology, University Hospital of Bern, Inselpital, Bern, Switzerland.

Conflicts of interest and sources of funding: none declared.

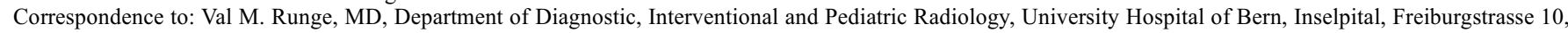
3010 Bern, Switzerland.

Copyright (C) 2015 Wolters Kluwer Health, Inc. All rights reserved.

ISSN: 0020-9996/15/5012-0811

DOI: $10.1097 /$ RLI.0000000000000229 